\title{
Domain size in systems with canted magnetization
}

\author{
Daniel Stickler, ${ }^{*}$ Robert Frömter, Holger Stillrich, Christian Menk, and Hans Peter Oepen \\ Universität Hamburg, Institut für Angewandte Physik, Jungiusstrasse 11, D-20355 Hamburg, Germany \\ Christian Gutt, Simone Streit-Nierobisch, Lorenz-M. Stadler, and Gerhard Grübel \\ Deutsches Elektronen-Synchrotron (DESY), Notkestrasse 85, D-22607 Hamburg, Germany \\ Carsten Tieg and Flora Yakhou-Harris \\ European Synchrotron Radiation Facility (ESRF), BP 220, F-38043 Grenoble CEDEX, France
}

(Received 30 September 2010; published 7 September 2011)

\begin{abstract}
The equilibrium domain size in ferromagnetic single-layer films with canted magnetization, as observed in the spin-reorientation transition, is analytically described. It is demonstrated that for canted magnetization in the case of narrow domain walls the size of the magnetic domains depends solely on the second-order anisotropy constant and not on the first-order anisotropy. The single-layer model is extended to the application to multilayers. An approximation for the domain size of multilayers is developed and used for the analysis of data obtained via soft x-ray holography. Our analysis, including the canting angle, allows for the determination of the relative anisotropy constants to second order.
\end{abstract}

DOI: 10.1103/PhysRevB.84.104412

PACS number(s): 75.70.Kw, 75.60.Ch, 75.70.Cn, 75.30.Gw

\section{INTRODUCTION}

The spin-reorientation transition in ultrathin films is in the focus of research since the successful realization of thin films with perpendicular easy axis of magnetization. ${ }^{1-3}$ The spin reorientation has been observed and studied in many systems, e.g., $\mathrm{Fe}$ on $\mathrm{Cu}(001)$ or $\mathrm{Ag}(001),{ }^{4-7} \mathrm{Co}$ on $\mathrm{Au}, \mathrm{Pd}$, and $\mathrm{Pt}$ (111) surface $^{8-13}$ and alloys. ${ }^{14,15}$ A comprehensive overview with further references to additional film systems can be found in Ref. 16. In most cases the driving parameter for a spin-reorientation transition is film thickness ${ }^{4,5,7,8,12,17,18}$ or temperature. ${ }^{6,19-21}$

One topic in research on spin reorientation in ultrathin films and multilayers is the formation of magnetic domains and its connection to the magnetic properties. As the domain structure in ultrathin films persists throughout the whole film depth, imaging of the domain pattern gives access to quantities like the total length of the domain walls and the domain size that are needed for a complete energy analysis. These quantities can then be used to determine the magnetic anisotropies when the system is driven toward spin reorientation. ${ }^{17}$ The domain structure close to and within the spin reorientation has been imaged in $\mathrm{Co} / \mathrm{Au}(111),{ }^{17,18,22} \mathrm{Fe} / \mathrm{Cu}(001),{ }^{20,21,23-26}$ $\mathrm{Fe} / \mathrm{Ni} / \mathrm{Cu}(001),{ }^{27,28}$ and $\mathrm{Co} / \mathrm{Pt}(111)^{29,30}$ utilizing different techniques.

In $\mathrm{Co} / \mathrm{Au}(111)$ the spin reorientation has been identified to proceed via the state of coexisting phases $\left(K_{2}<0\right) .{ }^{18,31} \mathrm{~A}$ domain collapse was found that could be described within first-order anisotropy approximation. ${ }^{17}$ Generally, a spinreorientation transition has to be described in second-order anisotropy approximation to compensate the apparent loss of anisotropy when the first-order anisotropy constant $K_{1}$ changes sign. A positive $K_{2}$ supports perpendicular magnetization alignment even for zero $K_{1}$. On the sign change of $K_{1}$ the magnetization starts to cant. Magnetic systems that exhibit this kind of spin reorientation are $\mathrm{Co} / \mathrm{Pt}$ multilayers. ${ }^{9,30,32,33} \mathrm{It}$ is well known that domains appear before and in the canting phase in $\mathrm{Co} / \mathrm{Pt}$ multilayers when $K_{1}$ is varied utilizing the
Co thickness as driving parameter. ${ }^{33-35}$ A collapse of domain sizes, like in $\mathrm{Co} / \mathrm{Au}(111)$, is not observed. On the contrary, a similar domain size of the order of $100-150 \mathrm{~nm}$ is frequently reported. ${ }^{29,30,36,37}$ In a first-order model the conclusion has been drawn from micromagnetic modeling of the observed domain patterns that the domain-wall energy stays almost constant in a certain thickness range. ${ }^{34}$

In this paper we demonstrate that in the case of narrow domain walls $K_{1}$ has no influence on the domain size in the canted phase. Assuming $180^{\circ}$ walls, it is demonstrated that the domain size is determined by $K_{2}$. To describe the evolution of the domain size in the canted phase as a function of magnetic anisotropy, in the first paragraph we derive an analytical model for single layers. In particular, we ask how the balance between the two competing energy contributions is affected as the magnetostatic energy is decreasing due to canting while the domain-wall energy is changing due to the anisotropy change. It is demonstrated that a lower limit for the obtainable domain size exists, which depends on the strength of the second-order anisotropy contribution.

To verify the results of experiments utilizing magnetic soft $\mathrm{X}$-ray Fourier-transform holography, ${ }^{38}$ in the next paragraph we work out an approximation of the model for the analysis of multilayers with a constant amount of active magnetic material. The results of domain structure investigation of $\mathrm{Co} / \mathrm{Pt}$ multilayers are finally analyzed using the approximation.

\section{DOMAIN SIZE AND MAGNETIZATION CANTING}

For ultrathin films with uniaxial perpendicular anisotropy the angular dependence of the free-energy density is described by a series expansion with respect to the angle $\theta$ to the easy axis, i.e., the surface normal. The free-energy density $f$ in second-order approximation is given by

$$
f=K_{1, \mathrm{eff}} \cdot \sin ^{2} \theta+K_{2} \cdot \sin ^{4} \theta .
$$


The effective first-order anisotropy constant $K_{1 \text {,eff }}$ contains three contributions: the surface/interface, the bulk, and the shape anisotropy. With the shape-anisotropy constant for ultrathin films $-\frac{\mu_{0}}{2} M_{\mathrm{s}}^{2}$ one obtains

$$
K_{1, \mathrm{eff}}=K_{\mathrm{V}}+\frac{K_{\mathrm{S}}+K_{\mathrm{I}}}{d}-\frac{\mu_{0}}{2} M_{\mathrm{s}}^{2} .
$$

$K_{\mathrm{V}}$ is the volume, $K_{\mathrm{S} / \mathrm{I}}$ the surface/interface contribution, and $d$ is the film thickness. The stability analysis of the energy density in second-order approximation leads to a $K_{1 \text {,eff }} / K_{2}$ phase diagram $^{31,39}$ that contains a canted phase separating the phases with perpendicular and in-plane orientations of the magnetization. The canted phase is stable for positive $K_{2}$ values and within the interval $-2 K_{2}<K_{1 \text {,eff }}<0$. It represents one of two possibilities for continuous transition from perpendicular to in-plane orientation of magnetization with a canting angle $\theta_{\mathrm{c}}$, to the surface normal, determined by

$$
\frac{K_{1, \text { eff }}}{2 K_{2}}=-\sin ^{2} \theta_{\mathrm{c}} .
$$

Alternatively, the spin-reorientation transition can proceed via a state of coexisting perpendicular and in-plane phases. ${ }^{31,39}$

Domains are created to lower the magnetostatic energy. The interplay of the latter with the total domain-wall energy leads to an average domain width, which was first analytically described for single layers by Kaplan and Gehring. ${ }^{40}$ The authors were able to derive an analytical approximation of the infinite series for the magnetostatic energy for thin films, which was given by Malek and Kambersky ${ }^{41}$ for the case of narrow domain walls. The validity of the analytical approximation was rigorously proven by Millev. ${ }^{42}$ In Ref. 40 the domain size $D$ as a function of magnetostatic and domain-wall energy $\gamma_{\mathrm{w}}$ is given by

$$
D\left(\gamma_{\mathrm{w}}, d\right)=d \cdot B \cdot \exp \left[\frac{\pi}{2} \cdot \frac{\gamma_{\mathrm{w}}}{E_{\mathrm{ms}} \cdot d}\right] .
$$

Here, $B$ is a constant that reflects the domain pattern geometry. $B$ is 0.955 for a stripe and 2.525 for a checkerboard pattern. ${ }^{40} E_{\mathrm{ms}}$ is the magnetostatic energy, which is $\frac{\mu_{0}}{2} M_{\mathrm{s}}^{2}$ in the case of a single domain film with perpendicular magnetization orientation.

The canting of magnetization causes a reduction of the magnetostatic energy, which depends on the canting angle

$$
E_{\mathrm{ms}, \mathrm{c}}\left(\theta_{\mathrm{c}}\right)=\frac{\mu_{0}}{2} M_{\mathrm{s}}^{2} \cos ^{2} \theta_{\mathrm{c}} .
$$

In the regime of magnetization canting the domain-wall energy is strongly affected by the second-order anisotropy contribution, which is always larger than $-0.5 K_{1, \text { eff }}$. An expression for the energy of $180^{\circ}$ domain walls in the canted phase has been given by Träuble et al. ${ }^{43}$ as

$$
\gamma_{\mathrm{w}, \mathrm{c}}=\frac{\pi}{2}\left(K_{1, \mathrm{eff}}+2 K_{2}\right) \sqrt{\frac{A}{K_{2}}}
$$

with $A$ for the exchange stiffness.

Equation (6) can be rearranged when introducing the canting angle $\theta_{\mathrm{c}}$ from Eq. (3),

$$
\gamma_{\mathrm{w}, \mathrm{c}}=\pi \sqrt{A K_{2}}\left\{1+\frac{K_{1, \mathrm{eff}}}{2 K_{2}}\right\}=\pi \sqrt{A K_{2}} \cos ^{2} \theta_{\mathrm{c}} .
$$

Inserting the magnetostatic [Eq. (5)] and the domain-wall energy [Eq. (7)] into the expression for the domain size [Eq. (4)], we obtain the analytical result for the equilibrium domain size in the phase of canted magnetization,

$$
D_{\mathrm{c}}\left(K_{2}, d\right)=d \cdot B \cdot \exp \left[\frac{\pi^{2}}{\mu_{0}} \cdot \frac{\sqrt{A K_{2}}}{M_{\mathrm{s}}^{2} \cdot d}\right] .
$$

Equation (8) represents an interesting and important result that is valid within the prerequisites of the model used for deriving the analytical formula for the magnetostatic energy. It reveals a distinct difference in the domain-size behavior compared to former investigations in the state of coexisting phases. The collapse of domain sizes on $K_{1 \text {,eff }}$ approaching zero is lifted. In the case of magnetization canting the domain size does not depend on $K_{1, \text { eff }}$ as long as $-K_{1 \text {,eff }}<K_{2}$ (small domain width). The domain size is determined by $K_{2}$, while $K_{1, \text { eff }}$ affects just the canting angle. Vice versa, it means that when crossing the canted phase the domain structure in the appropriate regime reveals whether or not $K_{2}$ changes.

Attention has to be paid when the thickness is used as driving parameter, since the domain size is also depending on thickness. A shrinking of domain sizes can appear and scales with $\exp (1 / d)$ on film thickness.

Hence from the evolution of the domain size a first clue on the evolution of the two anisotropy contributions can be obtained. The $K_{2}$ dependence can be calculated directly from the domain size while the canting angle must be analyzed for determination of $K_{1 \text {,eff }}$.

In the regime of perpendicular magnetization $\left(K_{1, \text { eff }} \geqslant 0\right)$ the energy for the $180^{\circ}$ domain walls is ${ }^{43}$

$$
\begin{aligned}
\gamma_{\mathrm{w}}= & 2 \sqrt{A K_{1, \mathrm{eff}}}\left[1+\left(\sqrt{\frac{K_{1, \mathrm{eff}}}{K_{2}}}+\sqrt{\frac{K_{2}}{K_{1, \mathrm{eff}}}}\right)\right. \\
& \left.\times \arcsin \left(\left[1+\frac{K_{1, \mathrm{eff}}}{K_{2}}\right]^{-1 / 2}\right)\right],
\end{aligned}
$$

which gives an identical form to the one in Ref. 44 .

For vanishing $K_{1 \text {,eff }}$ the last summand determines the wall energy and the energy approaches

$$
\gamma_{\mathrm{w}}=\pi \sqrt{A K_{2}}
$$

yielding the same domain size as given by Eq. (8) for the fixed thickness $d_{0}$, where the first-order anisotropy vanishes. As the domain-wall energy does not vanish for diminishingly small $K_{1, \text { eff }}$ the collapse of domain size will not be observed. The above considerations are exact in the case of a single-layer film as long as the analytical solution for the magnetostatic energy is also valid for magnetization canting and as long as the domain walls are narrow. The latter prerequisite inherently limits the range of validity of our model. As the domain-wall width $^{43}$

$$
\delta_{\mathrm{w}}=\frac{2 \pi \sqrt{A K_{2}}}{K_{1, \mathrm{eff}}+2 K_{2}}
$$

depends on $K_{2}$ and $K_{1, \text { eff }}$ the size increases when trespassing the canted phase toward the in-plane phase. The actual value of $K_{2}$ thus determines the applicability of our model. As a rule of thumb, the $K_{2}$ value gives a lower limit for the range of validity in $K_{1, \text { eff }}\left(-K_{1, \text { eff }}<K_{2}\right)$. For the Co/Pt multilayers the 
$K_{2}$ value is pretty large $\left(K_{2}=116 \mathrm{~kJ} / \mathrm{m}^{3}\right)$, which enlarges the range of $K_{1, \text { eff }}$ values and hence thicknesses where the model is reasonably applicable.

The influence of higher-order anisotropy contributions on the domain-wall energy and domain size has been considered in a publication before. ${ }^{45}$ The authors demonstrate that the domain-wall energy is finite at $K_{1 \text {,eff }}=0$ and that the collapse of domain size at $K_{1, \text { eff }}=0$ is prevented. ${ }^{45}$ For positive $K_{2}$ and changing sign of the first-order anisotropy the domain size has been theoretically considered in Ref. 46. As the authors use a very small $K_{2}$ value, they actually missed calculating the domain pattern in the canted phase. ${ }^{46}$ This is due to the fact that the main focus of the paper was on the investigation of the domain structure in the regime of coexisting phases.

\section{EXPERIMENT}

We have investigated Co/Pt multilayer films. The multilayers are deposited on a 100-nm-thick SiN membrane for imaging reasons (see below). To obtain good texture, a 5 -nm Pt buffer layer is deposited first by means of ion-beam sputtering utilizing an electron-cyclotron-resonance (ECR) ion source. ${ }^{33,47}$ On the seed layer a stack of $8 \times\left(\mathrm{Co}_{0.7 \mathrm{~nm}} / \mathrm{Pt}_{1.5 \mathrm{~nm}}\right)$ bilayers are deposited via magnetron sputtering. Finally the multilayer is capped by a 2-nm Pt layer. The detailed preparation conditions and the thickness dependence of $K_{\mathrm{V}}$ and $K_{\mathrm{I}}$ are published in Refs. 33 and 48.

The magnetization curves obtained via magneto-optical Kerr effect (MOKE) in perpendicular and in-plane magnetic fields are shown in Figs. 1(a) and 1(b). The hysteresis in perpendicular fields is well known and represents the situation that the multilayer decomposes into domains in vanishing fields. Details of the curves have been attributed to nucleation/annihilation and domain-wall propagation processes. ${ }^{49}$ The magnetization curve obtained with in-plane fields [Fig. 1(b)] has been shown to represent a system with canted magnetization. ${ }^{30}$ The latter magnetization curve can be used to determine the magnetic anisotropy by fitting $M(H)$ in second-order anisotropy approximation. ${ }^{33,50}$ We obtain $K_{1 \text { eff }}=(-31 \pm 8) \mathrm{kJ} / \mathrm{m}^{3}$ and $K_{2}=(116 \pm$ 12) $\mathrm{kJ} / \mathrm{m}^{3}$ utilizing $M_{\mathrm{s}}=1400 \mathrm{kA} / \mathrm{m}$ as the value for the saturation magnetization. ${ }^{51}$

For soft $x$-ray Fourier-transform holography imaging ${ }^{38}$ the membrane is covered by a thick Au film that is deposited on the backside. Into the opaque Au layer an object hole is milled via focused ion beam (FIB), which can be seen in Fig. 2. Around the larger object hole five reference holes are milled, which give five independent reference beams to interfere with the light passing the magnetic film above the object hole. The magnetic multilayer is deposited on the front (plane) side of the membrane after object-hole milling.

The multilayer is irradiated by ions through the membrane from the backside to modify the magnetic properties locally. The artificial tuning of magnetic properties ${ }^{52}$ or magnetic patterning ${ }^{53-55}$ by ions has been investigated in recent years. These investigations can be classified into two different topics: ion induced mixing and/or intermixing and ion implantation. Ion induced intermixing is typically used to manipulate the properties of interfaces, ${ }^{56}$ e.g., to alter exchange bias, ${ }^{57} \mathrm{GMR},{ }^{58,59}$ and/or interface anisotropy. ${ }^{60,61}$
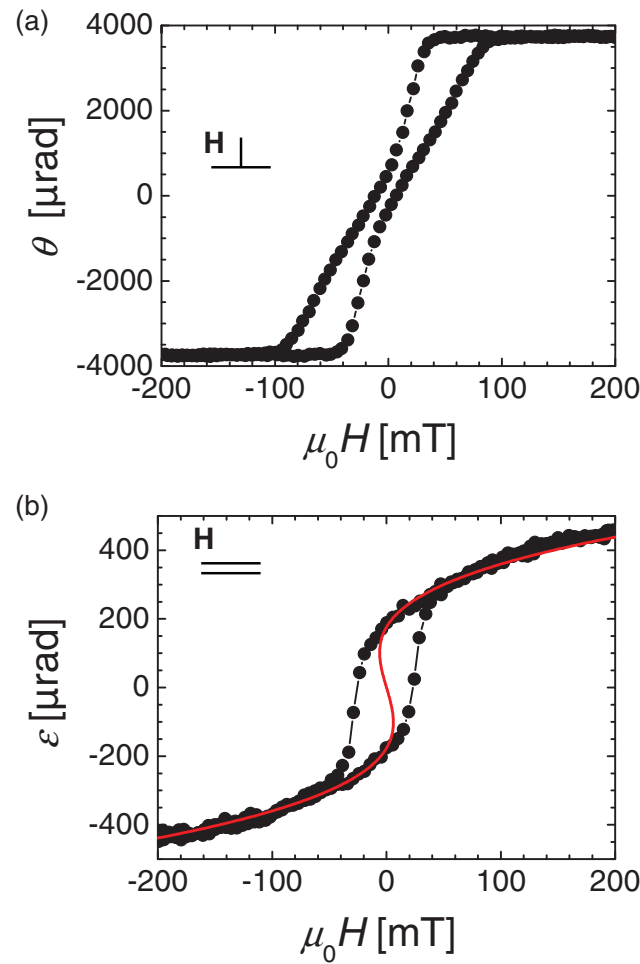

FIG. 1. (Color online) Kerr hysteresis loops of an $8 \times\left(\mathrm{Co}_{0.7 \mathrm{~nm}} / \mathrm{Pt}_{1.5 \mathrm{~nm}}\right)$ multilayer film. In (a) the Kerr rotation $\theta$ is given, obtained by sweeping a perpendicularly oriented field. The curve shows an easy-axis loop with a decay of the film into a multidomain state with almost vanishing remanence. In (b) the magnetization behavior (ellipticity $\varepsilon$ ) obtained in an in-plane magnetic field is given. The hysteresis exhibits a nonvanishing remanence, which is indicative of a canted magnetization (Ref. 30). The in-plane hysteresis is fitted taking into account the first- and second-order anisotropy constants according to Eq. (1) [solid line in (b)].

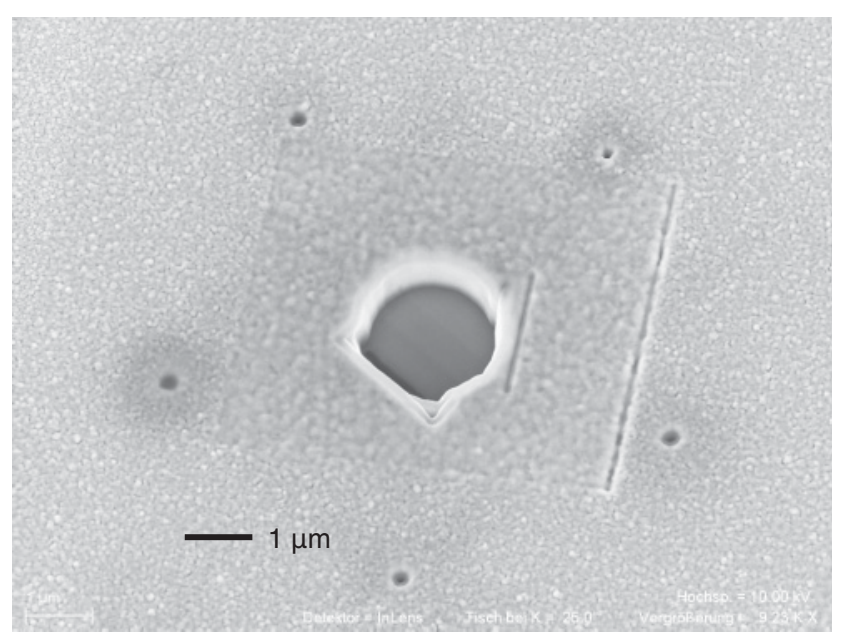

FIG. 2. (Color online) SEM micrograph (tilted view) of the holography sample. In the $1-\mu \mathrm{m}$-thick gold film that is deposited onto the $\mathrm{SiN}$ membrane an object hole with a diameter of $2 \mu \mathrm{m}$ has been milled. Five narrow reference holes are milled around the center of the object hole at a radius of $4.5 \mu \mathrm{m}$. 
In these experiments low area doses in the range of $10^{13}-10^{14}$ ions $/ \mathrm{cm}^{2}$ are used. Implantation of ions with a high area dose of $10^{15} \mathrm{ions} / \mathrm{cm}^{2}$ up to $10^{16} \mathrm{ions} / \mathrm{cm}^{2}$ can change, for example, the saturation magnetization, ${ }^{62}$ magnetic anisotropy, ${ }^{63,64}$ and strain. ${ }^{65}$ Usually the magnetic films are directly bombarded by ions. In our experiment the membrane acts as a moderator that reduces the detrimental effect of the ions on the magnetic properties. A considerable amount of the beam is absorbed or energy-reduced before reaching the multilayer film. ${ }^{37}$ This changes the regime from high dose/energy implantation and ion milling process to a low dose/energy intermixing experiment, but maintains the high spatial resolution of the FIB. In particular, in a certain ion-dose range the magnetic properties are altered while no multilayer material is removed. In this case the ions or displaced atoms that reach the multilayer cause a moderate modification of the multilayers. Most likely the interface and crystal structure are more or less affected. ${ }^{53,66,67}$ Utilizing the lateral resolution of FIB, three different doses are applied to three different areas of the multilayer, ${ }^{37}$ which can be seen in the scanning electron microscopy (SEM) micrograph in Fig. 3 (left) as horizontal stripes. A faint gray contrast can be recognized that makes the different areas visible. The area labeled (A) was not bombarded while regions (B)-(D) are irradiated by $2.5,25$, and $125 \times 10^{15}$ $\mathrm{Ga}^{+} / \mathrm{cm}^{2}$ in a multiscan application.

Using coherent soft x-ray illumination, the light from the reference holes and the object hole creates an interference pattern (hologram) on a charge-coupled device camera. ${ }^{68}$ Two different images at the $L_{3}$ absorption edge of Co $(778 \mathrm{eV})$ are taken with right- and left-circularly polarized light. The difference hologram is Fourier transformed and domain images become visible. ${ }^{37,38}$ The smallest reference hole used gives images with a high spatial resolution of $35 \mathrm{~nm}$.

The domain image is shown in Fig. 3 (right) where the four areas of different ion-dose application are indicated. Figure 3 reveals that the domain size becomes smaller with increasing dose. As the ion dose was too high in region (D), the magnetization is either rotated in plane or the ferromagnetic order has been destroyed. It is important to mention that

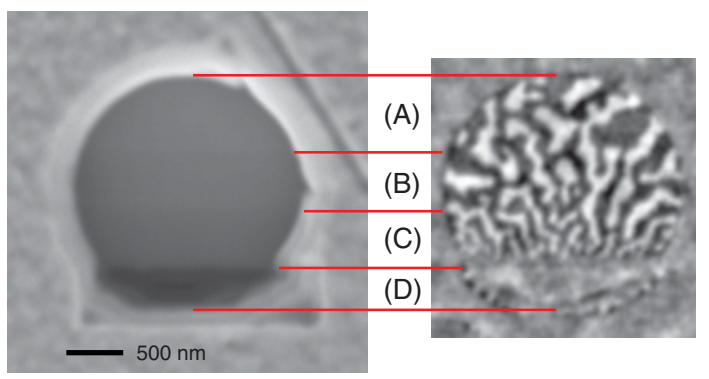

FIG. 3. (Color online) SEM micrograph and domain image of the same region of the sample. The four different areas in which different ion doses are applied are indicated by (A)-(D). In the different areas the following doses have been applied: $(\mathrm{A})=0,(\mathrm{~B})=2.5 \times$, $(C)=25 \times$, and $(D)=125 \times 10^{15} \mathrm{Ga}^{+} / \mathrm{cm}^{2}$. The size of magnetic domains changes with ion dose. In (D) the material has become either paramagnetic or the magnetization is lying in the film plane. A weak contrast in the left image reveals the regions of different dose application.

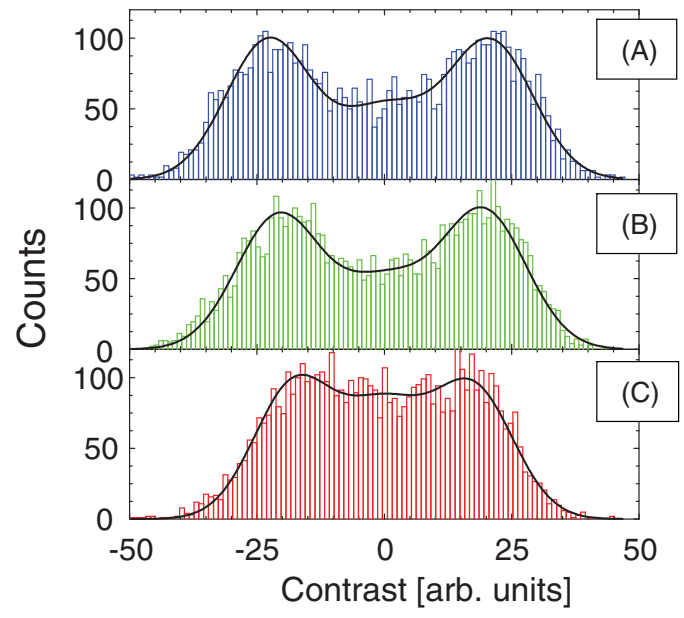

FIG. 4. (Color online) Histograms of the magnetic contrast extracted from the three areas exhibiting a magnetic signal [(A)-(C), see Fig. 3]. In (A) and (B) the contrast is well separated into peaks for black and white domains. In (C) the separation has become smaller while the contribution due the domain walls between the two peaks is increased.

the domain walls in all three regions appear as sharp lines, which means that the width is smaller than the resolution and considerably smaller than the domain size.

The domain pattern (Fig. 3) can be used to extract the mean domain size by a stereological procedure. ${ }^{44,69}$ The domain sizes in areas $(\mathrm{A})-(\mathrm{C})$ are $D_{(\mathrm{A})}=(121 \pm 4) \mathrm{nm}, D_{(\mathrm{B})}=(115 \pm$ $3) \mathrm{nm}$, and $D_{(\mathrm{C})}=(84 \pm 5) \mathrm{nm}$, respectively. As the hologram provides different states of magnetization in one image it can be used to compare quantitatively the magnetodichroic signals (magnetic contrast), which are proportional to the perpendicular magnetization component. Figure 4 displays the histogram of the contrast obtained for the three areas (A)-(C).

Each histogram was fitted by three Gaussian distributions. The outer maxima represent the domain magnetization while the inner distribution represents the portion of the images of and around domain walls. The distance between the two outer maxima has been taken as the magnetic contrast $(S)$. The relative change of contrast has been normalized with respect to area (A). We obtain a decrease of the domain contrast to (B) $(0.93 \pm 0.06)$ and $(C)(0.81 \pm 0.12)$.

The change of the magnetization component can be used to determine the change of canting angle. Hence such an image provides data on changes of domain structure and canting angle. The impact on the analysis is shown in the next section.

\section{ANALYSIS OF THE EXPERIMENTAL RESULTS}

To apply the above model to our multilayer systems with fixed composition we have to consider that the magnetostatic energy in a multilayer is different from a single layer. The magnetostatic self-energy in multilayers has been derived by Suna $^{70}$ for a stripe domain pattern. Draaisma and de Jonge $^{71}$ have included the effect of external fields into the description. Both papers assume infinitely sharp domain walls. The magnetostatic energy is given as an infinite series. 


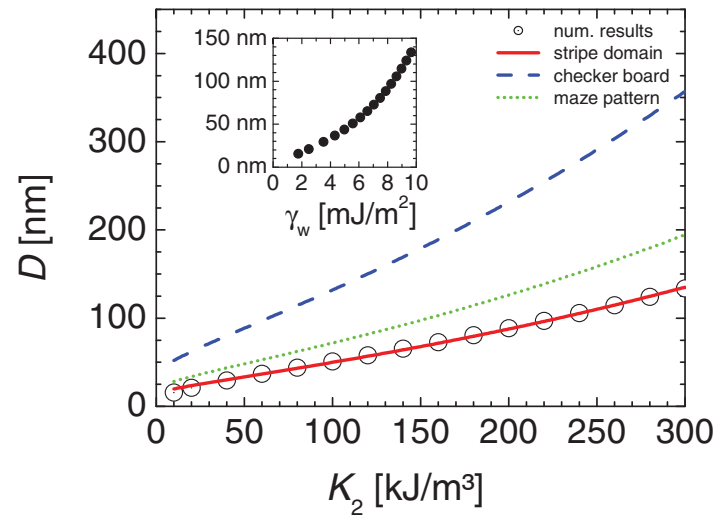

FIG. 5. (Color online) Calculated domain size as a function of $K_{2}$ (inset as a function of domain wall energy per unit area). The open dots show the minima of the sum of domain wall and stray field energy. The stray field energy was calculated using the formula for a stripe pattern given in Ref. 71. The domain size distribution of Eq. (12) has been fitted to the calculated values (red solid line). For comparison the size distribution for checkerboard and maze pattern are also plotted using appropriate assumptions for the geometrical factors (see text).

We use the numerical expression of Ref. 71 to calculate the magnetostatic energy as a function of domain size for a multilayer of fixed composition [e.g., $\left.\left(\mathrm{Co}_{0.7 \mathrm{~nm}} / \mathrm{Pt}_{1.5 \mathrm{~nm}}\right)_{8}\right]$. The infinite series of Eq. (2) in Ref. 71 was numerically calculated. The calculation was truncated at $n=5000$.

Next, the total energy, which is the sum of magnetostatic energy and the total domain-wall energy, is calculated as a function of the domain size. The minimum of the total energy is determined, which gives the equilibrium domain size as a function of $\gamma_{\mathrm{w}}$ (inset in Fig. 5). If the correction of the magnetostatic energy for the canting of magnetization is the same as in the single layer, both energy contributions will scale with $\cos ^{2} \theta_{c}$ and the equilibrium domain size is again a function of $K_{2}$ only. This allows us to plot the results of the numerical investigation (circles) versus $K_{2}$ (Fig. 5). These data points are fitted with an analytical expression that is similar to that for single layer films,

$$
D\left(K_{2}\right)=d_{\mathrm{eff}} \cdot B_{\mathrm{ML}} \cdot \exp \left[\frac{\pi^{2}}{2} \cdot \frac{\sqrt{A K_{2}}}{K_{\mathrm{d}} \cdot d_{\mathrm{eff}}}\right] .
$$

We have used used $A=31.4 \mathrm{pJ} / \mathrm{m},{ }^{72} d_{\text {eff }}$, and $B_{\mathrm{ML}}$ as fit parameters to obtain a good agreement in the span of $K_{2}$ values of interest. The resulting fit with the parameters $B_{\mathrm{ML}}=2.45$ \pm 0.04 and $d_{\text {eff }}=(5.22 \pm 0.07) \mathrm{nm}$ is also given in Fig. 5 . The total amount of Co in the multilayer is $5.6 \mathrm{~nm}$, while the fit gives a slightly smaller effective thickness.

Assuming the same ratio (2.64) of the geometry factors for stripe and checkerboard domain pattern as in single films we can also plot $D$ versus $K_{2}$ for a checkerboard (dashed line in Fig. 5).

As the ion bombardment does not remove material of the multilayer the composition is fixed. This allows us to assume that the magnetization is also conserved, so that we can apply the above multilayer formula [Eq. (12)] to the analysis of our experimental findings. At first we can conclude that the ion-beam mixing changes $K_{2}$ as the domain size is altered.
To quantify the change we have to eliminate the unknown geometry factor; neither a stripe nor a checkerboard pattern is found. For that reason we investigate the relative changes of domain size between two different stages of ion-beam mixing. We obtain from the ratio of domain sizes in equilibrium an expression for the change of the second-order anisotropy constant:

$$
K_{2, \mathrm{f}}=\left[\frac{M_{\mathrm{s}}^{2} d_{\mathrm{eff}}}{\sqrt{A}} \frac{\mu_{0}}{\pi^{2}} \ln \left(\frac{D_{\mathrm{f}}}{D_{\mathrm{i}}}\right)+\sqrt{K_{2, \mathrm{i}}}\right]^{2} .
$$

The indexes $\mathrm{i}$ and $\mathrm{f}$ stands for initial and final states, respectively.

Thus we can determine the changes in $K_{2}$ in the system on a scale well below the measurement accuracy of the MOKE setup we conventionally use to determine the magnetic properties. Absolute values are accessible when relying on the anisotropy values that were determined for the virgin film $\left(K_{2, \mathrm{i}}=116 \mathrm{~kJ} / \mathrm{m}^{3}\right)$. With $K_{2}$ we can also extract the $K_{1, \text { eff }}$ values utilizing the change in canting angle obtained from the magnetic contrast in the soft x-ray holography image. Again, we have to start with the values of the unperturbed film $\left(K_{1, \mathrm{eff}, \mathrm{i}}=-31 \mathrm{~kJ} / \mathrm{m}^{3}\right)$ and the observed change in magnetic contrast. With the ratio $R$ of the signals that is identical to the ratio of the perpendicular components of magnetization,

$$
R=\frac{M_{\mathrm{per}}^{\mathrm{f}}}{M_{\mathrm{per}}^{\mathrm{i}}}=\frac{\left(M_{\mathrm{s}} \cos \theta_{\mathrm{c}}\right)_{\mathrm{f}}}{\left(M_{\mathrm{s}} \cos \theta_{\mathrm{c}}\right)_{\mathrm{i}}}=\frac{S_{\mathrm{f}}}{S_{\mathrm{i}}},
$$

we can deduce the $K_{1, \text { eff }}$ value after ion mixing as

$$
K_{1, \mathrm{eff}, \mathrm{f}}=R^{2} \frac{K_{2, \mathrm{f}}}{K_{2, \mathrm{i}}}\left(2 K_{2, \mathrm{i}}+K_{1, \mathrm{eff}, \mathrm{i}}\right)-2 K_{2, \mathrm{f}} .
$$

For the smallest ion dose (B) we obtain $K_{1, \text { eff,f }}=(-55 \pm$ 14) $\mathrm{kJ} / \mathrm{m}^{3}$ and $K_{2, \mathrm{f}}=(108 \pm 15) \mathrm{kJ} / \mathrm{m}^{3}$, while the higher ion dose (C) changes the anisotropy values to $K_{1, \text { eff, } \mathrm{f}}=(-107 \pm$ 49) $\mathrm{kJ} / \mathrm{m}^{3}$ and $K_{2, \mathrm{f}}=(91 \pm 24) \mathrm{kJ} / \mathrm{m}^{3}$. The canting angle of magnetization changes from initially $\theta_{\mathrm{c},(\mathrm{A})}=21^{\circ}$ to (B) $\theta_{\mathrm{c},(\mathrm{B})}=30^{\circ}$ and $(\mathrm{C}) \theta_{\mathrm{c},(\mathrm{C})}=41^{\circ}$. The large error bars for the anisotropies in region $(\mathrm{C})$ are caused by a change of the domain pattern geometry from (A) (mazelike pattern) to a more stripelike pattern in (C). The problem is the prefactor in Eq. (12) and Eq. (8), which depends on the geometry of the domain structure and which is not known for the maze pattern. The ratio of the geometry factors for maze and stripe pattern can be extracted from measurements on the domain size for equivalent films in which both kinds of domain pattern were stabilized. In Ref. 36 the Co/Pt multilayer domain size in the maze pattern is found to be 1.44 times that of the domain stripe width. A similar factor has also been found for a 42 nm-thick GdFe single-layer film. ${ }^{73}$ This number gives us the scaling factor for $B_{\mathrm{ML}}$ in Eq. (14) from the stripe to the maze pattern. We have taken for the analysis the average of the values for maze and stripe and set the error to include both as lower and upper limits, which causes the large error margin. The evolution of the anisotropy values on the impact of ions is displayed in the $K_{1, \text { eff }} / K_{2}$ phase diagram in Fig. 6 . The magnetic system is more and more driven through the canted phase from vertical to in-plane orientation of magnetization upon increasing ion dose. 


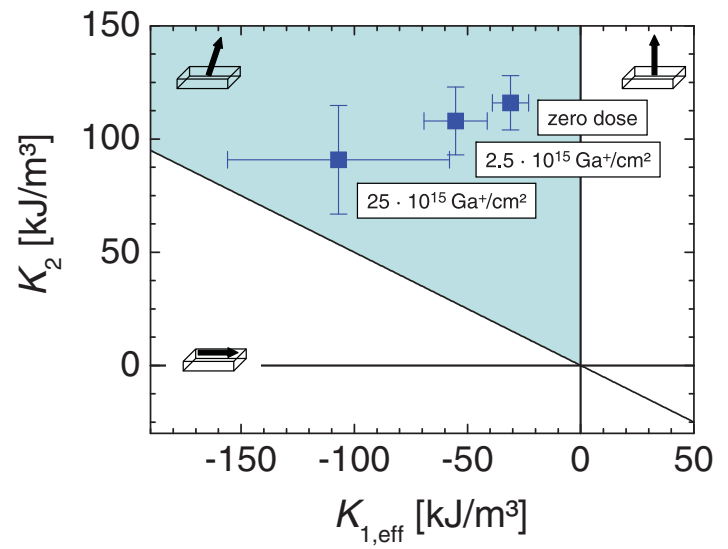

FIG. 6. (Color online) Phase diagram including the experimental results. The point that represents the nonirradiated part (A) is obtained from film measurements via MOKE. The other two points are extracted from the domain pattern utilizing the approximation of the model for multilayers. To prevent any further uncertainty, the relative changes to the film values have been determined (see text).

Question marks have to be put to the results obtained with the structure in region $\mathrm{C}$ as $\left(-K_{1, \text { eff }}\right)$ is larger than $K_{2}$. In that situation it is not clear whether the application of the formula for the domain size ${ }^{40}$ is allowed or not, although the domain-wall widths appear still reasonably narrow.

Up to the dose applied in range (C) only small amounts of the SiN layer are removed but no magnetic material. So, the changes measured are solely due to changes of the magnetic anisotropy. Low-energy ions can penetrate the multilayer and destroy to some amount the interface quality. ${ }^{56}$ This will surely contribute to the changes in the first-order anisotropy constant via reducing the interface contribution. The changes of the second-order anisotropy constant are most likely due to alteration of the Co quality. This hypothesis is based on earlier experiments where we could not identify any $K_{2}$ contribution that originates from the interfaces. ${ }^{33,48}$ One mechanism that might be responsible for the change of $K_{2}$ is the deformation of the SiN membrane by implanted Ga. The deformation of the membrane might cause strain in the metallic layer and thus changes of the volume anisotropy constants.

Finally we can calculate the different energy contributions based on the determined numbers. The magnetization canting causes a reduction of the magnetostatic energy by $13 \%$ in (C) compared to (A). The energy gain due to the decrease of domain size from 121 to $84 \mathrm{~nm}$ is around $3 \%$ without considering the additional domain-wall energy. Hence in the canted phase the reduction of magnetostatic energy is dominated by the canting itself.

\section{CONCLUSION}

We have presented an analytical formalism that describes the behavior of single-layer systems in the canted phase of the spin-reorientation transition in the limit of narrow $180^{\circ}$ domain walls. The most important finding is the fact that the domain size depends solely on the second-order anisotropy contribution and a collapse of domain sizes does not occur. The latter is a consequence of the domain-wall energy that merges into a constant value on the decrease of the effective first-order anisotropy constant. The model is valid in the canted phase for $-K_{1 \text {,eff }}<2 K_{2}$. For multilayers, we have worked out an analytical approximation that we have applied to results of recent domain pattern investigations of $\mathrm{Co} / \mathrm{Pt}$-multilayer films. We are able to determine relative changes of the magnetic anisotropy constants utilizing the domain-size measurements from images and the relative changes of canting angle.

\section{ACKNOWLEDGMENTS}

The authors like express their gratitude Yonko Millev for helpful discussions. We gratefully acknowledge the funding by DFG within SFB 668, as well as by BMBF via FSP 301 FLASH together with the Free and Hanseatic City of Hamburg via Grant No. HBFG1021-627 and from the Cluster of Excellence Nanospintronics funded by the Forschungs und Wissenschaftsstiftung Hamburg. *daniel.stickler@physnet.uni-hamburg.de

${ }^{1}$ U. Gradmann and J. Müller, Phys. Status Solidi B 27, 313 (1968).

${ }^{2}$ M. T. Johnson, P. J. H. Bloemen, F. J. A. denBroeder, and J. J. deVries, Rep. Prog. Phys. 59, 1409 (1996).

${ }^{3}$ J. Bland and B. Heinrich, Ultrathin Magnetic Structures I: An Introduction to the Electronic, Magnetic and Structural Properties (Springer, Berlin, 2005).

${ }^{4}$ N. C. Koon, B. T. Jonker, F. A. Volkening, J. J. Krebs, and G. A. Prinz, Phys. Rev. Lett. 59, 2463 (1987).

${ }^{5}$ C. Liu, E. R. Moog, and S. D. Bader, Phys. Rev. Lett. 60, 2422 (1988).

${ }^{6}$ D. P. Pappas, K.-P. Kämper, and H. Hopster, Phys. Rev. Lett. 64, 3179 (1990).

${ }^{7}$ J. Thomassen, F. May, B. Feldmann, M. Wuttig, and H. Ibach, Phys. Rev. Lett. 69, 3831 (1992).
${ }^{8}$ F. J. A. den Broeder, D. Kuiper, A. P. van de Mosselaer, and W. Hoving, Phys. Rev. Lett. 60, 2769 (1988).

${ }^{9}$ P. F. Carcia, J. Appl. Phys. 63, 5066 (1988).

${ }^{10}$ P. Bruno and J. P. Renard, Appl. Phys. A 49, 499 (1989).

${ }^{11}$ B. N. Engel, C. D. England, R. A. Van Leeuwen, M. H. Wiedmann, and C. M. Falco, Phys. Rev. Lett. 67, 1910 (1991).

${ }^{12}$ J.-W. Lee, J.-R. Jeong, S.-C. Shin, J. Kim, and S.-K. Kim, Phys. Rev. B 66, 172409 (2002).

${ }^{13}$ J.-W. Lee, J. Kim, S.-K. Kim, J.-R. Jeong, and S.-C. Shin, Phys. Rev. B 65, 144437 (2002).

${ }^{14}$ S.-K. Kim, J.-W. Lee, J.-R. Jeong, J. Kim, and S.-C. Shin, Appl. Phys. Lett. 79, 1652 (2001).

${ }^{15}$ S.-K. Kim, J. Kim, and S.-C. Shin, Phys. Rev. B 65, 094410 (2002).

${ }^{16}$ P. Jensen and K. Bennemann, Surf. Sci. Rep. 61, 129 (2006).

${ }^{17}$ M. Speckmann, H. P. Oepen, and H. Ibach, Phys. Rev. Lett. 75, 2035 (1995). 
${ }^{18}$ H. P. Oepen, M. Speckmann, Y. Millev, and J. Kirschner, Phys. Rev. B 55, 2752 (1997).

${ }^{19}$ A. Berger and H. Hopster, Phys. Rev. Lett. 76, 519 (1996).

${ }^{20}$ A. Enders, D. Peterka, D. Repetto, N. Lin, A. Dmitriev, and K. Kern, Phys. Rev. Lett. 90, 217203 (2003).

${ }^{21}$ D. Peterka, A. Enders, G. Haas, and K. Kern, Phys. Rev. B 66, 104411 (2002).

${ }^{22}$ R. Allenspach, M. Stampanoni, and A. Bischof, Phys. Rev. Lett. 65, 3344 (1990).

${ }^{23}$ R. Allenspach, J. Magn. Magn. Mater. 129, 160 (1994).

${ }^{24}$ E. Mentz, A. Bauer, T. Günther, and G. Kaindl, Phys. Rev. B 60, 7379 (1999).

${ }^{25}$ H. J. Choi, W. L. Ling, A. Scholl, J. H. Wolfe, U. Bovensiepen, F. Toyama, and Z. Q. Qiu, Phys. Rev. B 66, 014409 (2002).

${ }^{26}$ G. Meyer, T. Crecelius, A. Bauer, I. Mauch, and G. Kaindl, Appl. Phys. Lett. 83, 1394 (2003).

${ }^{27}$ J. Choi, J. Wu, C. Won, Y. Z. Wu, A. Scholl, A. Doran, T. Owens, and Z. Q. Qiu, Phys. Rev. Lett. 98, 207205 (2007).

${ }^{28}$ Y. Z. Wu, C. Won, A. Scholl, A. Doran, H. W. Zhao, X. F. Jin, and Z. Q. Qiu, Phys. Rev. Lett. 93, 117205 (2004).

${ }^{29}$ R. L. Stamps, L. Louail, M. Hehn, M. Gester, and K. Ounadjela, J. Appl. Phys. 81, 4751 (1997).

${ }^{30}$ R. Frömter, H. Stillrich, C. Menk, and H. P. Oepen, Phys. Rev. Lett. 100, 207202 (2008).

${ }^{31}$ Y. Millev and J. Kirschner, Phys. Rev. B 54, 4137 (1996).

${ }^{32}$ W. B. Zeper, F. J. A. M. Greidanus, P. F. Carcia, and C. R. Fincher, J. Appl. Phys. 65, 4971 (1989).

${ }^{33}$ H. Stillrich, C. Menk, R. Frömter, and H. P. Oepen, J. Appl. Phys. 105, 07 C308 (2009).

${ }^{34}$ L. Belliard, J. Miltat, V. Kottler, V. Mathet, C. Chappert, and T. Valet, J. Appl. Phys. 81, 5315 (1997).

${ }^{35}$ S.-B. Choe and S.-C. Shin, Phys. Rev. B 59, 142 (1999).

${ }^{36}$ O. Hellwig, G. P. Denbeaux, J. B. Kortright, and E. E. Fullerton, Physica B 336, 136 (2003).

${ }^{37}$ S. Streit-Nierobisch, D. Stickler, C. Gutt, L.-M. Stadler, H. Stillrich, C. Menk, R. Frömter, C. Tieg, O. Leupold, H. P. Oepen, and G. Grübel, J. Appl. Phys. 106, 083909 (2009).

${ }^{38}$ S. Eisebitt, J. Lüning, W. F. Schlotter, M. Lörgen, O. Hellwig, W. Eberhardt, and J. Stöhr, Nature (London) 432, 885 (2004).

${ }^{39}$ H. Casimir, J. Smit, U. Enz, J. Fast, H. Wijn, E. Gorter, A. Duyvesteyn, J. Fast, and J. de Jong, J. Phys. Radium 20, 360 (1959).

${ }^{40}$ B. Kaplan and G. Gehring, J. Magn. Magn. Mater. 128, 111 (1993).

${ }^{41}$ Z. Malek and V. Kambersky, Czech. J. Phys. 8, 416 (1958).

${ }^{42}$ Y. Millev, J. Phys.: Condens. Matter 8, 3671 (1996).

${ }^{43}$ H. Träuble, O. Boster, H. Kronmüller, and A. Seeger, Phys. Status Solidi B 10, 283 (1965).

${ }^{44}$ A. Hubert and R. Schäfer, Magnetic Domains (Springer-Verlag, Berlin, Heidelberg, 2009).

${ }^{45}$ A. Maziewski, V. Zablotskii, and M. Kisielewski, Phys. Status Solidi A 189, 1001 (2002).

${ }^{46}$ V. Zablotskii, W. Stefanowicz, and A. Maziewski, J. Appl. Phys. 101, 113904 (2007).

${ }^{47}$ M. Wellhöfer, M. Weißenborn, R. Anton, S. Pütter, and H. P. Oepen, J. Magn. Magn. Mater. 292, 345 (2005).

${ }^{48}$ H. Stillrich, C. Menk, R. Frömter, and H. P. Oepen, J. Magn. Magn. Mater. 322, 1353 (2010).
${ }^{49}$ J. E. Davies, O. Hellwig, E. E. Fullerton, G. Denbeaux, J. B. Kortright, and K. Liu, Phys. Rev. B 70, 224434 (2004).

${ }^{50} \mathrm{~W}$. Sucksmith and J. E. Thompson, Proc. R. Soc. London, Ser. A 225, 362 (1954).

${ }^{51}$ M. B. Stearns, Landolt-Börnstein: Magnetic Properties: Magnetic Properties of Metals: 3d, 4d and 5d Elements, Alloys and Compounds Group III, Vol. 19, Pt. a (Springer-Verlag, Berlin, 1986).

${ }^{52}$ J. Fassbender, D. Ravelosona, and Y. Samson, J. Phys. D 37, R179 (2004).

${ }^{53}$ J. Fassbender and J. McCord, J. Magn. Magn. Mater. 320, 579 (2008).

${ }^{54}$ C. Chappert, H. Bernas, F. J., V. Kottler, J.-P. Jamet, Y. Chen, E. Cambril, T. Devolder, F. Rousseaux, V. Mathet, and H. Launois, Science 280, 1919 (1998).

${ }^{55}$ J. Gierak, D. Mailly, P. Hawkes, R. Jede, L. Bruchhaus, L. Bardotti, B. Pravel, P. Malinon, A. Perez, R. Hyndman, J.-P. Jamet, J. Ferre, A. Mougin, C. Chappert, V. Mathet, P. Warin, and J. Chapman, Appl. Phys. A 80, 187 (2005).

${ }^{56}$ T. Devolder, Phys. Rev. B 62, 5794 (2000).

${ }^{57}$ S. Blomeier, D. McGrouther, R. O’Neill, S. McVitie, J. Chapman, M. Weber, B. Hillebrands, and J. Fassbender, J. Magn. Magn. Mater. 290-291, 731 (2005).

${ }^{58}$ Z. Guo, D. You, J. Qiu, K. Li, and Y. Wu, Solid State Commun. 120, 459 (2001).

${ }^{59}$ E. Kerr, S. van Dijken, R. Langford, and J. Coey, J. Magn. Magn. Mater. 290-291, 124 (2005).

${ }^{60}$ C. Vieu, J. Gierak, H. Launois, T. Aign, P. Meyer, J. P. Jamet, J. Ferre, C. Chappert, T. Devolder, V. Mathet, and H. Bernas, J. Appl. Phys. 91, 3103 (2002).

${ }^{61}$ J. Ferré, T. Devolder, H. Bernas, J. P. Jamet, V. Repain, M. Bauer, N. Vernie, and C. Chappert, J. Phys. D 36, 3103 (2003).

${ }^{62}$ C. Faulkner, D. Atkinson, D. Allwood, and R. Cowburn, J. Magn. Magn. Mater. 319, 9 (2007).

${ }^{63}$ J. McCord, T. Gemming, L. Schultz, J. Fassbender, M. O. Liedke, M. Frommberger, and E. Quandt, Appl. Phys. Lett. 86, 162502 (2005).

${ }^{64}$ J. McCord, I. Mönch, J. Fassbender, A. Gerber, and E. Quandt, J. Phys. D 42, 055006 (2009).

${ }^{65}$ N. Martin, J. McCord, A. Gerber, T. Strache, T. Gemming, I. Mönch, N. Farag, R. Schäfer, J. Fassbender, E. Quandt, and L. Schultz, Appl. Phys. Lett. 94, 062506 (2009).

${ }^{66}$ R. Hyndman, P. Warin, J. Gierak, J. Ferre, J. N. Chapman, J. P. Jamet, V. Mathet, and C. Chappert, J. Appl. Phys. 90, 3843 (2001).

${ }^{67}$ J. Fassbender, A. Mücklich, K. Potzger, and W. Möller, Nucl. Instrum. Methods Phys. Res. B 248, 343 (2006).

${ }^{68}$ S. Eisebitt, M. Lörgen, W. Eberhardt, J. Lüning, and J. Stöhr, Appl. Phys. A 80, 921 (2005).

${ }^{69}$ R. Bodenberger and A. Hubert, Phys. Status Solidi A 44, K7 (1977).

${ }^{70}$ A. Suna, J. Appl. Phys. 59, 313 (1986).

${ }^{71}$ H. J. G. Draaisma and W. J. M. de Jonge, J. Appl. Phys. 62, 3318 (1987).

${ }^{72}$ G. Shirane, V. J. Minkiewicz, and R. Nathans, J. Appl. Phys. 39, 383 (1968).

${ }^{73}$ J. Miguel, J. F. Peters, O. M. Toulemonde, S. S. Dhesi, N. B. Brookes, and J. B. Goedkoop, Phys. Rev. B 74, 094437 (2006). 